University of Nebraska - Lincoln

DigitalCommons@University of Nebraska - Lincoln

January 1993

Relative photoionization and photodetachment cross sections for particular fine-structure transitions with application to $\mathrm{Cl}$ $3 s$-subshell photoionization

Cheng Pan

University of Nebraska - Lincoln

Anthony F. Starace

University of Nebraska-Lincoln, astarace1@unl.edu

Follow this and additional works at: https://digitalcommons.unl.edu/physicsstarace

Part of the Physics Commons

Pan, Cheng and Starace, Anthony F., "Relative photoionization and photodetachment cross sections for particular fine-structure transitions with application to $\mathrm{Cl} 3$ s-subshell photoionization" (1993). Anthony F. Starace Publications. 48.

https://digitalcommons.unl.edu/physicsstarace/48

This Article is brought to you for free and open access by the Research Papers in Physics and Astronomy at DigitalCommons@University of Nebraska - Lincoln. It has been accepted for inclusion in Anthony F. Starace Publications by an authorized administrator of DigitalCommons@University of Nebraska - Lincoln. 


\title{
Relative photoionization and photodetachment cross sections for particular fine-structure transitions with application to $\mathrm{Cl} 3 s$-subshell photoionization
}

\author{
Cheng Pan \\ Department of Physics and Astronomy, The University of Nebraska-Lincoln, Lincoln, Nebraska 68588-0111 \\ Anthony F. Starace* \\ Joint Institute for Laboratory Astrophysics, University of Colorado and National Institute of Standards and Technology, \\ Boulder, Colorado 80309-0440
}

(Received 18 May 1992; revised manuscript received 21 August 1992)

\begin{abstract}
The relative photoionization cross section for starting from a particular fine-structure level of the initial state of an atomic system and leading to a particular fine-structure level of the residual ion is presented for the case of atomic systems having one open subshell using a simple theoretical model in which emphasis is placed on the analysis of geometrical effects. This model parametrizes photoionization cross sections in terms of $L S$-dependent, single-configuration dynamical amplitudes, unlike previous treatments which assume $L S$-independent transition amplitudes from the start. In special cases our general formulas are shown to reduce to those of previous workers. We highlight photoionization of inner $s$ subshells in open-shell atoms and show that when the total angular momenta for the initial atomic and final ionic fine-structure levels differ by more than $\frac{1}{2}$ then such transitions are quasiforbidden. This prediction is tested in detail for the case of $\mathrm{Cl} 3 s$-subshell photoionization by comparison of our single-configuration results with the multiconfiguration, eigenchannel $R$-matrix results of Robicheaux and Greene [Phys. Rev. A (to be published)]. All of our results apply also to photoionization of open-shell positive ions or photodetachment of open-shell negative ions.
\end{abstract}

PACS number(s): $32.80 . \mathrm{Fb}$

\section{INTRODUCTION}

Increasingly many photoionization experiments are being done with high-resolution photoelectron spectrometry [1]. Experiments of this type can provide the partial cross section for a transition from a particular finestructure level of the initial atomic state to a particular fine-structure level of the residual ion. Such partial cross sections offer more severe tests of theoretical approximation methods than do the cross sections measured without regard to individual fine-structure levels. However, it is important to distinguish geometric from dynamic effects, particularly since the geometric effects alone can result in intensity distributions that are quite different from those expected for a statistically averaged atomic state. In this paper we provide a geometric analysis of the relative intensities to be expected for the partial photoionization (as well as photodetachment) cross sections for particular fine-structure transitions. This analysis permits one to determine which experimentally measured transitions are influenced strongly by dynamical effects.

Theoretical efforts to disentangle geometrical effects from dynamical effects in atomic photoprocesses have a long history. The initial stimuli appear to have been the measurements of Lineberger and co-workers on finestructure transitions in photodetachment of negative ions in the early $1970 \mathrm{~s}[2,3]$. Rau and Fano $[4,5]$ were able to provide interpretations of the observed [2,3] branching ratios for photodetachment of negative ions using a pri- marily geometrical analysis. The key point of their analysis is that in the region of space where photoprocesses occur, near the origin, the transition amplitudes are unaffected by the generally small spin-orbit and other relativistic interactions which lead to fine structure. Those weak interactions affect primarily the kinetic energy of the electron at asymptotic distances. Hence the relative magnitudes for particular fine-structure transitions may be calculated analytically using only the geometric (i.e., angular momentum) properties of the transitions. The process of photodetachment is much simpler than that of photoionization since near the threshold for the former process only a single photoelectron orbital angular momentum dominates. In particular, the photodetachment processes considered by Rau and Fano $[4,5]$ involved primarily $s$-wave photoelectrons, which simplifies the theoretical analysis significantly.

While the general case of photoionization was sketched in the review of Rau [5], it was not analyzed in detail [6]. However, the work of Fano and Rau on photodetachment stimulated several other researchers to examine the general case of atomic photoionization [7-11]. All of these works make the additional assumption from the start that the dynamical transition amplitudes are independent of the orbital and spin angular momentum quantum numbers relevant to the transition under consideration except for the orbital angular momentum of the photoelectron. In the case that $L S$-coupled single configurations are used to represent the initial and fi- 
nal states, all of these authors obtain the same results. While the assumption of $L S$-independent transition amplitudes is not generally a good one, it permits theory to predict the relative cross sections for photoinduced finestructure transitions completely analytically and hence provides experimenters with benchmarks against which to compare.

With the advent of several new synchrotron light sources around the world, which will permit experimentalists to study a host of additional photoionization processes, including especially inner-shell photoionization processes, a more detailed theoretical analysis of the geometrical aspects of the general photoionization process is called for. Futhermore, Fano [12] has emphasized the utility of a theoretical framework for atomic photoprocesses "within which branching ratios can be discussed without relying from the start upon a restrictive model." Our general photoionization formulas for finestructure transitions represent an attempt at providing such a less restrictive theoretical framework. While for simplicity we have assumed a single-configuration representation of the initial and final states, we have retained the $L S$-coupling dependence of the dynamical (electric dipole) transition amplitudes. Our general formulas may, of course, be used to parametrize experimental partial cross sections in terms of the $L S$-dependent radial dipole amplitudes and photoelectron phase shifts. In special cases, our results for the partial cross sections for particular fine-structure transitions are shown to reduce to those obtained by others for photodetachment $[4,5]$ and photoionization [7-11]. The utility of our more general analysis is highlighted by a detailed examination of photoionization of inner $s$ subshells in open-shell atoms. In the approximation that the transition amplitudes are $L S$ independent, we show that certain transitions are forbidden. (Namely, those for which the initial atomic and final ionic total angular momentum quantum numbers $J_{0}$ and $J_{c}$ differ by more than $\frac{1}{2}$.) Our more general formula in which the transition amplitudes are $L S$ dependent shows these transitions to be allowed. The implication of these two formulas is that there are unsuspected cancellation effects between different transition amplitudes contributing to these particular fine-structure transitions. Even though both our $L S$-dependent results and our $L S$-independent results assume a single initial and final configuration for the atom or ion, these predictions of unsuspected quasiforbidden transitions are shown to have validity for the case of $\mathrm{Cl} 3 s$-subshell photoionization, for which recent experimental and more detailed theoretical results are available.

In Sec. II we present our general expressions for the partial cross sections for fine-structure transitions for an arbitrary atom or ion having a single open subshell. Excitations of inner and outer subshell electrons are treated separately. In Sec. III we examine three special cases of our general formulas: The case of closed-shell atoms or ions, the case of photodetachment of negative ions, and the case of term-independent transition amplitudes, all three of which permit analytic predictions of relative partial cross sections. In Sec. IV we examine in detail the case of photoionization of inner $s$ subshells of open- shell atoms. In Sec. V we consider in detail the case of Cl $3 s$-subshell photoionization and compare our results with recent experimental and other theoretical work. Finally, in Sec. VI we discuss various aspects of our results and present our conclusions.

\section{PARTIAL CROSS SECTIONS FOR FINE-STRUCTURE TRANSITIONS}

We consider in this paper the partial cross sections for photoionization of an open-shell atom having $N$ electrons in the open subshell. There are two cases: one may photoionize an electron from the open subshell or from one of the closed subshells. The transitions for each of these two cases may be written as follows:

$$
\begin{aligned}
\mathcal{A} n_{0} l_{0}^{N}\left(L_{0} S_{0} J_{0}\right) & +\gamma \rightarrow \mathcal{A}^{+} n_{0} l_{0}^{N-1}\left(L_{c} S_{c} J_{c}\right)+e^{-} \\
\mathcal{A} n_{0} l_{0}^{4 l_{0}+2} n_{1} l_{1}^{N}( & \left.L_{0} S_{0} J_{0}\right)+\gamma \\
& \rightarrow \mathcal{A}^{+} n_{0} l_{0}^{4 l_{0}+1} n_{1} l_{1}^{N}\left(L_{c} S_{c} J_{c}\right)+e^{-}
\end{aligned}
$$

The theoretical model we use to describe each of these two transitions has the following features. First, both the initial atomic state and the final ionic state are assumed to be describable by a single $L S$ multiplet of a single electronic configuration. The final state of the ion-electron system is described by the coupled product of the wave function of the ionic core with a distorted wave function with definite momentum $\mathbf{k}$ (as well as a spin function) for the photoelectron. This final-state wave function can be expanded in terms of wave functions with definite total angular momentum $J$ using the $J_{c} j$ coupling scheme, where $j$ results from the coupling of each partial wave $(l)$ and the spin $\left(\frac{1}{2}\right)$ of the photoelectron.

We follow Briggs's procedures [13] for calculating the electric dipole transition matrix element for the transitions in Eqs. (1) and (2), as discussed in more detail below. The partial cross sections are obtained by summing over final magnetic substates, averaging over initial magnetic substates, and, finally, summing over the final quantum numbers $j$ (of the photoelectron) and $J$ (of the ion-electron system). We discuss each of the two cases in Eqs. (1) and (2) in turn.

\section{A. Photoionization of the open subshell}

For incident light polarized with polarization vector $\hat{\boldsymbol{\epsilon}}$, the partial cross section for the transition given in Eq. (1) is (in a.u.) [14] 


$$
\sigma\left(J_{0}, J_{c}\right)=\frac{4 \pi^{2} \omega}{c\left[J_{0}\right]} \sum_{M_{0}, M, l, j, J}\left|\left\langle n_{0} l_{0}^{N} L_{0} S_{0} J_{0} M_{0}|D| n_{0} l_{0}^{N-1}\left(L_{c} S_{c}\right) J_{c}\left(l \frac{1}{2}\right) j J M-\right\rangle\right|^{2}
$$

where $D=\sum_{i} \hat{\epsilon} \cdot r_{i}=\sum_{i} \sum_{q=-1}^{+1} \epsilon_{q}^{*} r_{i q}^{(1)}$ is the electric dipole operator (expressed respectively in vector and spherical tensor form), $\left[J_{0}\right] \equiv 2 J_{0}+1, \omega$ is the photon energy, and $c$ is the speed of light. The minus sign in the final-state ket indicates that the final-state wave functions satisfy incoming-wave boundary conditions [14]. The transition matrix element in Eq. (3) may be written as a sum of products of three factors:

$$
\begin{aligned}
\left\langle n_{0} l_{0}^{N} L_{0} S_{0} J_{0} M_{0}|D| n_{0} l_{0}^{N-1}\left(L_{c} S_{c}\right) J_{c}\left(l \frac{1}{2}\right) j J M-\right\rangle= & \sum_{L S}\left\langle n_{0} l_{0}^{N} L_{0} S_{0}\left\|\sum_{j} r_{j}^{(1)}\right\| n_{0} l_{0}^{N-1}\left(L_{c} S_{c}\right) \epsilon l L S-\right\rangle \\
& \times\left[(-1)^{L_{0}+S_{0}-M_{0}}\left(\left[J_{0}\right][J]\right)^{1 / 2} \sum_{q} \epsilon_{q}^{*}\left(\begin{array}{ccc}
J & 1 & J_{0} \\
M & q-M_{0}
\end{array}\right)\left\{\begin{array}{ccc}
J & 1 & J_{0} \\
L_{0} & S_{0} & L
\end{array}\right\}\right] \\
& \times\left(\left(L_{c} l\right) L\left(S_{c} \frac{1}{2}\right) S \mid\left(L_{c} S_{c}\right) J_{c}\left(l \frac{1}{2}\right) j\right)_{J} .
\end{aligned}
$$

The first factor is the reduced $L S$-coupling transition matrix element obtained by following Briggs's rules (i)-(xi) in Sec. 6.1 of Ref. [13]. This factor has been evaluated in the review by Starace [15]:

$$
\begin{aligned}
\left\langle n_{0} l_{0}^{N} L_{0} S_{0}\left\|\sum_{j} r_{j}^{(1)}\right\| n_{0} l_{0}^{N-1} L_{c} S_{c} \epsilon l L S-\right\rangle= & \delta_{S_{0} S}\left(\left[l_{0}\right][l]\left[L_{0}\right][L] N\right)^{1 / 2}\left(l_{0}^{N} L_{0} S_{0}\left\{\mid l_{0}^{N-1} L_{c} S_{c}\right)(-1)^{L_{0}+L_{c}}\right. \\
& \times\left\{\begin{array}{ccc}
l_{0} & 1 & l \\
L & L_{c} & L_{0}
\end{array}\right\}\left(\begin{array}{ccc}
l_{0} & 1 & l \\
0 & 0 & 0
\end{array}\right)\left\langle n_{0} l_{0}|r| \epsilon l\right\rangle_{L} \exp \left(-i \phi_{\epsilon \ell}^{L}\right),
\end{aligned}
$$

where $\left\langle n_{0} l_{0}|r| \epsilon l\right\rangle_{L}$ is the one-electron radial dipole matrix element between the radial wave function of the initial-state orbital $n_{0} l_{0}$ and the radial wave function of a partial-wave $l$ of the final state of the electron, normalized to a $\delta$ function in energy. $\phi_{\epsilon \ell}^{L}$ is the sum of the phase shift of the photoelectron with respect to a Coulomb wave and the Coulomb phase $\sigma_{\ell}$ [14]. (In the case of photodetachment, $\phi_{\epsilon \ell}^{L}$ is the phase shift with respect to a plane wave.) In general both the radial matrix element and the phase shift depend on the $L S$-coupling quantum numbers of the transition. We have indicated the dependence on $L$ explicitly; the dependence on $L_{c}, S_{c}$, and $S$ is implicit. The second factor in Eq. (4) comprises the fine-structuredependent factors given by rule (xii) of Ref. [13], as shown in Ref. [16]. Finally, the third factor in Eq. (4) is a recoupling coefficient, which serves to convert the final state from $L S J$ coupling to $J_{c} j J$ coupling [17]:

$$
\begin{aligned}
\left(\left(L_{c} l\right) L\left(S_{c} \frac{1}{2}\right) S \mid\left(L_{c} S_{c}\right) J_{c}\left(l \frac{1}{2}\right) j\right)_{J} & \\
= & \left([L][S]\left[J_{c}\right][j]\right)^{1 / 2}\left\{\begin{array}{lll}
L_{c} & l & L \\
S_{c} & \frac{1}{2} & S \\
J_{c} & j & J
\end{array}\right\} .
\end{aligned}
$$

The partial cross section $\sigma\left(J_{0}, J_{c}\right)$ in Eq. (3) may be evaluated simply. Substituting Eqs. (4)-(6) into Eq. (3), the sums over $M_{0}$ and $M$ may be carried out using the orthogonality relation for $3 j$ symbols [18]. The sum over $j$ may be performed using a standard sum rule for the product of two $9 j$ symbols [19]; the result is a $12 j$ symbol of the second kind. Finally, the sum over $J$ involves the product of two $6 j$ symbols and the $12 j$ symbol of the second kind just mentioned. This sum is easily performed graphically using the two simplest rules for graphical addition of angular momenta [20]. Our general result finally is

$$
\begin{aligned}
& \sigma\left(J_{0}, J_{c}\right)=\frac{4 \pi^{2}}{3 c} \omega\left[\ell_{0}\right]\left[L_{0}\right]\left[S_{0}\right]\left[J_{c}\right] N\left(\ell_{0}^{N} L_{0} S_{0}\left\{\mid \ell_{0}^{N-1} L_{c} S_{c}\right)^{2}\right. \\
& \times \sum_{\ell}[\ell] \sum_{L}[L] \sum_{L^{\prime}}\left[L^{\prime}\right]\left\langle n_{0} \ell_{0}|r| \epsilon \ell\right\rangle_{L}\left\langle n_{0} \ell_{0}|r| \epsilon \ell\right\rangle_{L^{\prime}} \exp i\left(\phi_{\epsilon \ell}^{L^{\prime}}-\phi_{\epsilon \ell}^{L}\right) \\
& \times\left\{\begin{array}{ccc}
L_{0} & L_{c} \ell_{0} \\
\ell & 1 & L
\end{array}\right\}\left\{\begin{array}{ccccc}
L_{0} & L_{c} \ell_{0} \\
\ell & 1 & L^{\prime}
\end{array}\right\}\left[\begin{array}{llllllll}
L_{c} & S_{c} & S_{0} & L_{0} & L \\
& J_{c} & \frac{1}{2} & & J_{0} & & 1 & \ell \\
L_{c} & S_{c} & S_{0} & & L_{0} & L^{\prime}
\end{array}\right] .
\end{aligned}
$$

In Eq. (7) the last symbol is a $15 j$ symbol of the second kind, which has the convenient property that it may be expressed as a single sum over five $6 j$ symbols [21]: 


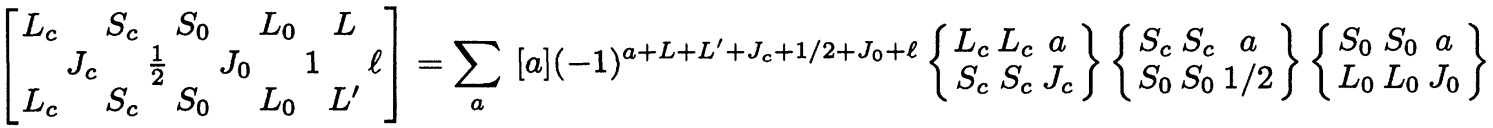

$$
\begin{aligned}
& \times\left\{\begin{array}{lll}
L_{0} & L_{0} & a \\
L^{\prime} & L & 1
\end{array}\right\}\left\{\begin{array}{lll}
L & L^{\prime} & a \\
L_{c} & L_{c} & \ell
\end{array}\right\} .
\end{aligned}
$$

\section{B. Photoionization of a closed-shell in an open-shell atom}

The partial cross section $\sigma\left(J_{0}, J_{c}\right)$ for the process in Eq. (2) (in which an electron in the $n_{0} l_{0}$ closed subshell is photoionized in an atom having an open $n_{1} l_{1}$ subshell) is calculated analogously to that for the process in Eq. (1). In fact, the only difference from the calculation described above for Eq. (1) is that the reduced $L S$-coupling transition matrix element is different from that in Eq. (5) [22]:

$$
\begin{aligned}
\left\langle n_{0} l_{0}^{4 l_{0}+2} n_{1} l_{1}^{N} L_{0} S_{0}\left\|\sum_{j} r_{j}^{(1)}\right\| n_{0} l_{0}^{4 l_{0}+1} n_{1} l_{1}^{N} L_{c} S_{c} \epsilon l L S-\right\rangle= & \delta_{S S_{0}}(-1)^{N+L_{0}+L_{c}}\left(\frac{\left[l_{0}\right][l]\left[L_{c}\right]\left[S_{c}\right][L]}{\left[S_{0}\right]}\right)^{1 / 2}\left\{\begin{array}{cc}
l_{0} & 1 \\
L & L_{c} \\
L_{0}
\end{array}\right\} \\
& \times\left(\begin{array}{ccc}
l_{0} & 1 & l \\
0 & 0 & 0
\end{array}\right)\left\langle n_{0} l_{0}|r| \epsilon l\right\rangle_{L} \exp \left(-i \phi_{\epsilon \ell}^{L}\right) .
\end{aligned}
$$

The general result for the partial cross section $\sigma\left(J_{0}, J_{c}\right)$ is

$$
\begin{aligned}
& \sigma\left(J_{0}, J_{c}\right)=\frac{4 \pi^{2} \omega}{3 c}\left[\ell_{0}\right]\left[L_{0}\right]\left[L_{c}\right]\left[S_{c}\right]\left[J_{c}\right] \sum_{\ell}[\ell] \sum_{L}[L] \sum_{L^{\prime}}\left[L^{\prime}\right]\left\langle n_{0} \ell_{0}|r| \epsilon \ell\right\rangle_{L}\left\langle n_{0} \ell_{0}|r| \epsilon \ell\right\rangle_{L^{\prime}} \exp i\left(\phi_{\epsilon \ell}^{L^{\prime}}-\phi_{\epsilon \ell}^{L}\right)
\end{aligned}
$$

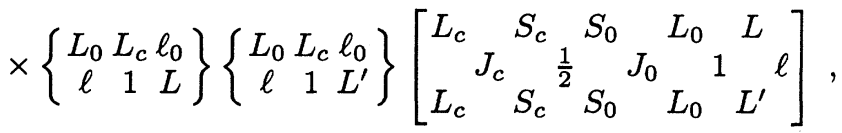

where the $15 j$ symbol is again evaluated according to Eq. (8).

\section{SPECIAL CASES}

Due to the summations over $\ell, L$, and $L^{\prime}$ in the general partial cross section formulas given in Eqs. (7) and (10), the dynamical and geometric factors in these equations are tangled together. We briefly point out here three special cases in which geometric and dynamical factors become disentangled.

\section{A. Cases in which one or more angular momenta of the initial and/or the core state are zero (e.g., closed-shell atoms)}

When one or more of the quantum numbers $L_{c}, S_{c}$, $S_{0}$, and/or $L_{0}$ are zero, then the summation index $a$ in Eq. (8) is also zero. This makes the evaluation of the $15 j$ symbol trivial, and, in particular, makes the cross sections in Eqs. (7) and (10) proportional to $2 J_{c}+1$. For example, when $L_{0}=0$, Eq. (7) becomes

$$
\begin{aligned}
\sigma\left(J_{0}, J_{c}\right)_{L_{0}=0}=\frac{4 \pi^{2} \omega}{3 c} \sum_{\ell} & {[l]\left(\begin{array}{ccc}
l_{0} & 1 & l \\
0 & 0 & 0
\end{array}\right)^{2}\left\langle n_{0} l_{0}|r| \epsilon l\right\rangle_{L=1}^{2} } \\
& \times \delta_{L_{c} l_{0}} \delta_{S_{0} J_{0}} \frac{\left[J_{c}\right] N}{\left[L_{c}\right]\left[S_{c}\right]} \\
& \times\left(l_{0}^{N} L_{0} S_{0}\left\{\mid l_{0}^{N-1} L_{c} S_{c}\right)^{2} .\right.
\end{aligned}
$$

The case of closed-shell atoms is included in Eq. (11); in that case one uses $N=4 l_{0}+2$ and $S_{0}=J_{0}=0$.

\section{B. Photodetachment, particularly of a $p$-subshell electron}

In general, Eqs. (7) and (10) simplify near the threshold for a photodetachment process according to Wigner's threshold law [23], i.e., the partial cross sections are dominated by those dynamical amplitudes having the lowest allowed value of the photoelectron's orbital angular momentum $\ell$. If one were to ignore all other dynamical amplitudes, Eqs. (7) and (10) would simplify only to the extent that there would no longer be a sum over $\ell$, whose single value would be known. However, the summations over $L$ and $L^{\prime}$ would still prevent one from obtaining a separation of dynamical and geometric factors. 
The case of photodetachment of a $p$-subshell electron, such as the case of $\mathrm{S}^{-}$photodetachment treated by Rau and Fano $[4,5]$, does, however, permit a disentangling of dynamical and geometric factors. A $p$-subshell electron may be detached as either an $s$ wave or a $d$ wave according to electric dipole selection rules. However, near threshold, only amplitudes having $\ell=0$ matter [23]. When $\ell$ is set equal to zero in the last $6 j$ symbol of Eq. (8), one obtains the result that $L$ and $L^{\prime}$ are restricted to the value $L_{c}$. Hence in the general Eqs. (7) and (10) all three sums over $\ell, L$, and $L^{\prime}$ collapse resulting in a complete separation of dynamical and geometric factors.

More specifically, consider the special case of Eq. (7) in which the open $p$ subshell of an arbitrary negative ion is photodetached. When we set $\ell=0$ in Eq. (8), the $15 j$ symbol reduces to a single sum over four different $6 j$ symbols. However, this sum equals a $12 j$ symbol of the second kind [24], whose symmetry properties [25] permit it to be rewritten as a single sum over the product of the squares of only two different $6 j$ symbols. We obtain thereby from Eq. (7) the following result:

$$
\begin{aligned}
\sigma_{\ell=0}( & \left.J_{0}, J_{c}\right) \\
= & \frac{4 \pi^{2}}{3 c} \omega\left[L_{0}\right]\left[S_{0}\right]\left[J_{c}\right] N\left(p^{N} L_{0} S_{0}\left\{\mid p^{N-1} L_{c} S_{c}\right)^{2}\right. \\
& \times\left\langle n_{0} \ell_{0}|r| \epsilon s\right\rangle_{L=L_{c}}^{2} \delta_{\ell_{0} 1} \\
& \times \sum_{\lambda}[\lambda]\left\{\begin{array}{ccc}
L_{c} & S_{0} & \lambda \\
\frac{1}{2} & J_{c} & S_{c}
\end{array}\right\}^{2}\left\{\begin{array}{ccc}
L_{c} & S_{0} & \lambda \\
J_{0} & 1 & L_{0}
\end{array}\right\}^{2},
\end{aligned}
$$

where $\lambda$ is a summation index. For the case of photodetachment of $\mathrm{S}^{-}$one has $S_{0}=\frac{1}{2}, L_{0}=1, L_{c}=1$, and $S_{c}=1$. Substituting these quantum numbers into Eq. (12) gives essentially the same formula obtained by Rau [26].

\section{Approximation of term-independent dynamical amplitudes}

If the radial matrix elements in our general expressions in Eqs. (7) and (10) are assumed independent of the orbital angular momenta $L$ and $L^{\prime}$, then one may proceed to carry out the summations over $L$ and $L^{\prime}$ analytically. Such term independence is typical of central potential model and configuration-average Hartree-Fock calculations. The summations over $L$ and $L^{\prime}$ proceed by using Eq. (8) and the orthogonality relation for $6 j$ symbols [27]. There remains a single summation of the four remaining $6 \ddot{j}$ symbols over the summation variable $a$ [cf. Eq. (8)]. This summation equals a $12 j$ symbol of the second kind [24]. Using the symmetry properties of this $12 j$ symbol [25], however, one may rewrite the sum as being over the product of the squares of only two $6 j$ symbols.

The partial photoionization cross section for process (1), given in general by Eq. (7), becomes in the termindependent approximation

$$
\begin{aligned}
& \sigma\left(J_{0}, J_{c}\right)=\frac{4 \pi^{2} \omega}{3 c} \sum_{\ell}[l]\left(\begin{array}{ccc}
l_{0} & 1 & l \\
0 & 0 & 0
\end{array}\right)^{2}\left\langle n_{0} l_{0}|r| \epsilon l\right\rangle^{2}\left[L_{0}\right]\left[S_{0}\right]\left[J_{c}\right] N\left(l_{0}^{N} L_{0} S_{0}\left\{\mid l_{0}^{N-1} L_{c} S_{c}\right)^{2}\right. \\
& \times \sum_{\lambda}[\lambda]\left\{\begin{array}{ccc}
L_{c} & S_{0} & \lambda \\
\frac{1}{2} & J_{c} & S_{c}
\end{array}\right\}^{2}\left\{\begin{array}{lll}
L_{c} & S_{0} & \lambda \\
J_{0} & l_{0} & L_{0}
\end{array}\right\}^{2}
\end{aligned}
$$

where $\lambda$ is a summation index. Similarly, the partial photoionization cross section for process (2), given in general by Eq. (10), becomes in the term-independent approximation

$$
\sigma\left(J_{0}, J_{c}\right)=\frac{4 \pi^{2} \omega}{3 c} \sum_{\ell}[l]\left(\begin{array}{ccc}
l_{0} & 1 & l \\
0 & 0 & 0
\end{array}\right)^{2}\left\langle n_{0} l_{0}|r| \epsilon l\right\rangle^{2}\left[L_{c}\right]\left[S_{c}\right]\left[J_{c}\right] \sum_{\lambda}[\lambda]\left\{\begin{array}{ccc}
L_{c} & S_{0} & \lambda \\
\frac{1}{2} & J_{c} & S_{c}
\end{array}\right\}^{2}\left\{\begin{array}{ccc}
L_{c} & S_{0} & \lambda \\
J_{0} & l_{0} & L_{0}
\end{array}\right\}^{2} .
$$

Equation (13) is equivalent to the single-configuration, $L S$-coupling, term-independent results of others [28].

\section{PHOTOIONIZATION OF INNER $s$ SUBSHELLS}

Photoionization (or photodetachment) of an inner $s$ subshell of an open-shell atom is a particular case of process (2) in which $\ell_{0}=0$. Our general $L S$-coupling result for this case in Eq. (10) becomes

$$
\begin{aligned}
\sigma\left(J_{0}, J_{c}\right)=\frac{4 \pi^{2}}{3 c} \omega\left[L_{c}\right]\left[S_{c}\right]\left[J_{c}\right] \delta_{L_{c} L_{0}} \sum_{L, L^{\prime}} & (-1)^{L+L^{\prime}}[L]\left[L^{\prime}\right]\left\langle n_{0} s|r| \epsilon p\right\rangle_{L}\left\langle n_{0} s|r| \epsilon p\right\rangle_{L^{\prime}} \exp i\left(\phi_{\epsilon p}^{L^{\prime}}-\phi_{\epsilon p}^{L}\right) \\
\times & {\left[\begin{array}{llllllll}
L_{0} & S_{c} & S_{0} & L_{0} & L \\
& J_{c} & 1 / 2 & J_{0} & & 1 & 1 \\
L_{0} & & S_{c} & S_{0} & L_{0} & L^{\prime}
\end{array}\right] }
\end{aligned}
$$


TABLE I. Relative cross section ${ }^{\mathrm{a}} \sigma\left(J_{0}, J_{c}\right)$ for the process $\mathcal{A} n s^{2} n p^{N}\left({ }^{2 S_{0}+1} L_{0} J_{0}\right)+\gamma \rightarrow \mathcal{A}^{+} n s n p^{N}\left({ }^{2 S_{c}+1} L_{c J_{c}}\right)+e^{-}$ $(N=1$ or 5$)$.

\begin{tabular}{|c|c|c|c|c|}
\hline \multirow{2}{*}{$\begin{array}{l}\text { Initial state } \\
{ }^{2 S_{0}+1} L_{0 J_{0}}\end{array}$} & \multicolumn{4}{|c|}{ Residual ion } \\
\hline & ${ }^{3} P_{2}$ & ${ }^{3} P_{1}$ & ${ }^{3} P_{0}$ & ${ }^{1} P_{1}$ \\
\hline${ }^{2} P_{3 / 2}$ & 5 & 1 & 0 & 2 \\
\hline${ }^{2} P_{1 / 2}$ & 0 & 4 & 2 & 2 \\
\hline
\end{tabular}

${ }^{a}$ Units are chosen so that all of the results have integer values. This choice is different from those used in other tables.

where the $15 j$ symbol is again evaluated according to Eq. (8).

In the approximation that the dynamic amplitudes (i.e., the radial matrix elements and phase shifts) are independent of $L$ and $L^{\prime}$, Eq. (15) becomes equal to Eq. (14) with $\ell_{0}=0$, i.e.,

$\sigma\left(J_{0}, J_{c}\right)=\frac{4 \pi^{2} \omega}{3 c} \delta_{L_{c} L_{0}}\left\langle n_{0} s|r| \epsilon p\right\rangle^{2}\left[S_{c}\right]\left[J_{c}\right]\left\{\begin{array}{ccc}L_{0} & S_{0} & J_{0} \\ \frac{1}{2} & J_{c} & S_{c}\end{array}\right\}^{2}$.

One observes immediately that the $6 j$ symbol in Eq. (16) is zero unless the following triangular relation is satisfied:

$$
\left|J_{0}-\frac{1}{2}\right| \leq J_{c} \leq J_{0}+\frac{1}{2} .
$$

However, according to the more general expression in Eq. (15), nonzero cross sections $\sigma\left(J_{0}, J_{c}\right)$ are permitted even for values of $J_{0}$ and $J_{c}$ which do not satisfy the triangular relation in Eq. (17). This is so because no such triangular relation is implied by the $15 j$ symbol in Eq. (15), as may be verified by examination of either Eq. (8) or the graphical representation of the $15 j$ symbol of the second kind [29].

One concludes, therefore, that when photoionizing inner $s$ subshells of open-shell atoms, fine-structure transitions for which $J_{0}$ and $J_{c}$ differ by more than $\frac{1}{2}$ (and for which $\delta_{L_{c} L_{0}}=1$ ) are quasiforbidden. The fact that the term-independent approximation for $\sigma\left(J_{0}, J_{c}\right)$ is zero when $J_{0}$ and $J_{c}$ differ by more than $\frac{1}{2}$ indicates an unsuspected strong cancellation among the $L S$-dependent am- plitudes in the more general expression for $\sigma\left(J_{0}, J_{c}\right)$ given in Eq. (15). Measurement or calculation of $\sigma\left(J_{0}, J_{c}\right)$ for such transitions thus provides a sensitive measure of the term dependence of the dynamical amplitudes.

To illustrate the case of Eq. (16), we present in Tables I-III the relative fine-structure cross sections for photoionization of inner $s$ subshells in open $p$-subshell atoms,

$\mathcal{A} n_{0} s^{2} n_{1} p^{N}\left(L_{0} S_{0} J_{0}\right)+\gamma \rightarrow \mathcal{A}^{+} n_{0} s n_{1} p^{N}\left(L_{0} S_{c} J_{c}\right)+e^{-}$

where $N=1-5$. (A similar set of tables for photoionization of the open $p$ subshell has been given in Ref. [10].) One observes immediately from the tables that a large number of fine-structure transitions for which $\delta_{L_{c} L_{0}}=1$ are quasiforbidden.

\section{APPLICATION TO Cl 3s-SUBSHELL PHOTOIONIZATION}

While the usefulness of the purely geometrical partial cross section formula in Eq. (13) for predicting the fine-structure branching ratios for transitions from the outer open subshell of an atom or ion is well-known [5,711], the corresponding predictions for inner $s$-subshells in open-shell atoms or ions may be viewed more skeptically. For this reason, we examine the predictions of our formulas in Eqs. (15) and (16) for process (2) for the particular case of atomic chlorine. This case was chosen because there exist experimental data [30] for this process and also because Robicheaux and Greene have recently carried out multiconfiguration, eigenchannel R-matrix calculations for photoionization of both the $3 p$-subshell [31] and $3 s$-subshell [32] of atomic chlorine. Reference [32] does not contain plots of the fine-structure partial cross sections; however, Robicheaux has provided us their data so we can make a comparison of our results with theirs in this paper. In order for us to present the predictions of our Eq. (15), we have carried out $L S$-dependent HartreeFock (HF) calculations for $\mathrm{Cl} 3 s$-subshell photoionization. The predictions of our Eq. (16) are obtained from Table I.

Our relative cross section results for the process

$$
\mathrm{Cl} 3 s^{2} 3 p^{5}\left({ }^{2} P_{J_{0}}\right)+\gamma \rightarrow \mathrm{Cl} 3 s 3 p^{5}\left({ }^{3} P_{J_{c}}\right)+e^{-}
$$

TABLE II. Relative cross section ${ }^{\text {a }} \sigma\left(J_{0}, J_{c}\right)$ for the process $\mathcal{A} n s^{2} n p^{N}\left({ }^{2 S_{0}+1} L_{0} J_{0}\right)$

\begin{tabular}{|c|c|c|c|c|c|c|c|c|}
\hline \multirow{2}{*}{$\begin{array}{l}\text { Initial state } \\
{ }^{2 S_{0}+1} L_{0 J_{0}}\end{array}$} & \multicolumn{8}{|c|}{ Residual ion ${ }^{2 S_{c}+1} L_{c J_{c}}$} \\
\hline & ${ }^{4} P_{5 / 2}$ & ${ }^{4} P_{3 / 2}$ & ${ }^{4} P_{1 / 2}$ & ${ }^{2} P_{3 / 2}$ & ${ }^{2} P_{1 / 2}$ & ${ }^{2} D_{5 / 2}$ & ${ }^{2} D_{3 / 2}$ & ${ }^{2} S_{1 / 2}$ \\
\hline${ }^{3} P_{2}$ & 27 & 3 & 0 & 15 & 0 & 0 & 0 & 0 \\
\hline${ }^{3} P_{1}$ & 0 & 25 & 5 & 5 & 10 & 0 & 0 & 0 \\
\hline${ }^{3} P_{0}$ & 0 & 0 & 30 & 0 & 15 & 0 & 0 & 0 \\
\hline${ }^{1} D_{2}$ & 0 & 0 & 0 & 0 & 0 & 27 & 18 & 0 \\
\hline${ }^{1} S_{0}$ & 0 & 0 & 0 & 0 & 0 & 0 & 0 & 45 \\
\hline
\end{tabular}
$+\gamma \rightarrow \mathcal{A}^{+} n s n p^{N}\left({ }^{2 S_{c}+1} L_{c J_{c}}\right)+e^{-}(N=2$ or 4$)$.

a Units are chosen so that all of the results have integer values. This choice is different from those used in other tables. 
TABLE III. Relative cross section ${ }^{\mathrm{a}} \sigma\left(J_{0}, J_{c}\right)$ for the process $\mathcal{A} n s^{2} n p^{3}\left({ }^{2 S_{0}+1} L_{0} J_{0}\right)$ $+\gamma \rightarrow \mathcal{A}^{+} n s n p^{3}\left({ }^{2 S_{c}+1} L_{c J_{c}}\right)+e^{-}$.

\begin{tabular}{|c|c|c|c|c|c|c|c|c|c|c|}
\hline \multirow{2}{*}{$\begin{array}{l}\text { Initial state } \\
{ }^{2 S_{0}+1} L_{0 J_{0}}\end{array}$} & \multicolumn{10}{|c|}{ Residual ion ${ }^{2 S_{c}+1} L_{c J_{c}}$} \\
\hline & ${ }^{5} S_{2}$ & ${ }^{3} S_{1}$ & ${ }^{3} D_{3}$ & ${ }^{3} D_{2}$ & ${ }^{3} D_{1}$ & ${ }^{1} D_{2}$ & ${ }^{3} P_{2}$ & ${ }^{3} P_{1}$ & ${ }^{3} P_{0}$ & ${ }^{1} P_{1}$ \\
\hline${ }^{4} S_{3 / 2}$ & 15 & 9 & 0 & 0 & 0 & 0 & 0 & 0 & 0 & 0 \\
\hline${ }^{2} D_{5 / 2}$ & 0 & 0 & 14 & 4 & 0 & 6 & 0 & 0 & 0 & 0 \\
\hline${ }^{2} D_{3 / 2}$ & 0 & 0 & 0 & 9 & 9 & 6 & 0 & 0 & 0 & 0 \\
\hline${ }^{2} P_{3 / 2}$ & 0 & 0 & 0 & 0 & 0 & 0 & 15 & 3 & 0 & 6 \\
\hline${ }^{2} P_{1 / 2}$ & 0 & 0 & 0 & 0 & 0 & 0 & 0 & 12 & 6 & 6 \\
\hline
\end{tabular}

a Units are chosen so that all of the results have integer values. This choice is different from those used in other tables.

are presented in Fig. 1 for $J_{0}=\frac{3}{2}$ and in Fig. 2 for $J_{0}=\frac{1}{2}$. The purely geometrical predictions of our Eq. (16), which assumes $L S$-independent dynamical amplitudes, are indicated by the horizontal solid lines (cf. Table I). These predictions agree surprisingly well with those obtained using our more general Eq. (15), in which the $L S$-dependent amplitudes and phase shifts are calculated in $\mathrm{HF}$ approximation. Finally, these single-configuration predictions agree very well with the multiconfiguration, eigenchannel $R$-matrix results of Robicheaux and Greene [32], except of course in the vicinity of autoionizing resonances. This agreement is all the more remarkable since they find that the ${ }^{3} P^{o}$ state of the ion only contains $71 \%$ $3 s 3 p^{5}$ and $29 \%$ other configurations. In conclusion, it appears that the prediction of the quasiforbidden transitions $J_{0}=\frac{3}{2} \rightarrow J_{c}=0$ (cf. Fig. 1) and $J_{0}=\frac{1}{2} \rightarrow J_{c}=2$ (cf. Fig. 2) by our Eq. (16) does give a reliable indicator of otherwise unsuspected cancellation among the amplitudes contributing to these processes. This is true not only in the case of our more general Eq. (15) but also in the case of the multiconfiguration, eigenchannel $R$-matrix calculations [32].

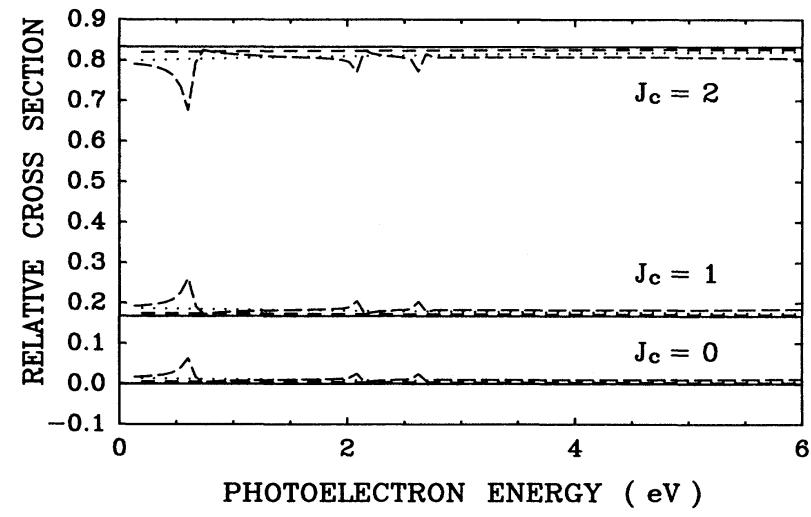

FIG. 1. Relative cross sections for the transitions $\mathrm{Cl}$ $3 s^{2} 3 p^{5}\left({ }^{2} P_{3 / 2}\right)+\gamma \rightarrow \mathrm{Cl} 3 s 3 p^{5}\left({ }^{3} P_{J_{c}}\right)+e^{-}$in three levels of approximation. Solid lines: purely geometrical predictions of Eq. (16) (cf. Table I). Short-dashed (dotted) lines: LSdependent HF predictions using Eq. (15) in dipole length (velocity) approximation. Long-dashed lines: multiconfiguration, eigenchannel $R$-matrix results of Robicheaux and Greene [32].
Further evidence of the usefulness of Eq. (16) is provided by a recent experiment [30]. This experiment was unable to discern any signal for the two quasiforbidden transitions indicated in Table I (as well as in Figs. 1 and 2) for $\mathrm{Cl} 3 s$-subshell photoionization. It thus confirms that the quasiforbidden transitions are very weak. However, this experiment also determined a branching ratio of 0.31 for the ratio ${ }^{3} P_{1}:{ }^{3} P_{2}$ of the partial cross sections for process (19) for photoionization from the $J_{0}=\frac{3}{2}$ initial state at a photon energy of $29.2 \mathrm{eV}$ [30]. This compares with our purely geometrical prediction of 0.20 , our $L S$ dependent $\mathrm{HF}$ prediction of 0.21 , and the eigenchannel $R$-matrix result of Robicheaux and Greene of 0.23 . The reason for this significant discrepancy of experiment with all three theoretical predictions is unclear.

Finally, we consider now the branching ratio for the ${ }^{3} P_{2,1,0}$ and ${ }^{1} P_{1}$ final states. Specifically, consider the photoionization of the chlorine ground state, i.e.,

$\mathrm{Cl} 3 s^{2} 3 p^{5}\left({ }^{2} P_{J_{0}}\right)+\gamma \rightarrow \mathrm{Cl} 3 s 3 p^{5}\left({ }^{2 S_{c}+1} P_{J_{c}}\right)+e^{-}$,

where $S_{c}=0$ or 1 . The ratio we are interested in is the triplet: singlet branching ratio, i.e.,

$$
\frac{\sum_{J_{c}} \sigma\left({ }^{2} P_{J_{0}} \rightarrow{ }^{3} P_{J_{c}}\right)}{\sigma\left({ }^{2} P_{J_{0}} \rightarrow{ }^{1} P_{1}\right)}=3 .
$$

The value 3 in Eq. (21) may be verified from Table I for

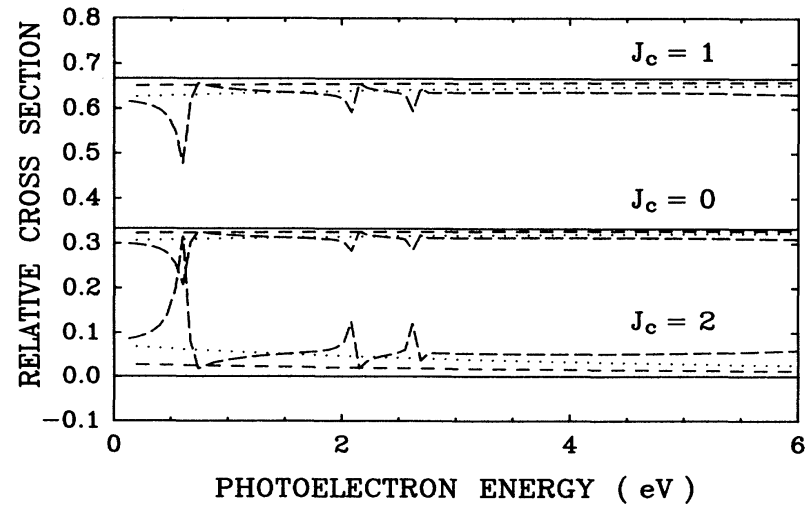

FIG. 2. Relative cross sections for the transitions $\mathrm{Cl}$ $3 s^{2} 3 p^{5}\left({ }^{2} P_{1 / 2}\right)+\gamma \rightarrow \mathrm{Cl} 3 s 3 p^{5}\left({ }^{3} P_{J_{c}}\right)+e^{-}$in three levels of approximation, as specified in Fig. 1 . 
either initial state, $J_{0}=\frac{3}{2}$ or $J_{0}=\frac{1}{2}$. In fact, one may show very easily from either of our general formulas in Eq. (7) or Eq. (10) that $\sum_{J_{c}} \sigma\left(J_{0}, J_{c}\right)$ is independent of $J_{0}$ [33]. For inner $s$ subshells the result of this sum over $J_{c}$ [using Eq. (10)] is proportional to the final-state core spin multiplicity, $2 S_{c}+1$. When the dynamical amplitudes are assumed to be independent of $S_{c}$, this fact then explains the value 3 given on the right in Eq. (21).

Figure 3 shows the prediction of Eq. (21), based upon use of Eq. (16), that ${ }^{3} P_{2,1,0}:{ }^{1} P_{1}$ is $0.75: 0.25$ and compares this result with predictions based upon use of our Eq. (15), which are obtained by calculating the amplitudes and phase shifts in an $L S$-dependent HF approximation [34]. One sees that these two single-configuration predictions give nearly the same results. The predictions of the multiconfiguration, eigenchannel $R$-matrix calculations [32], however, differ significantly, having a ratio for ${ }^{3} P_{2,1,0}:{ }^{1} P_{1}$ of about 0.88:0.12. (Only eigenchannel $R$ matrix results for the $J_{0}=\frac{3}{2}$ initial state are shown; results for the $J_{0}=\frac{1}{2}$ initial state, however, are very similar.) The origin of this discrepancy is most likely the large configuration mixing found for the ${ }^{1} P^{\circ}$ final ionic state: it is only $40 \% 3 s 3 p^{5}$ and $60 \%$ other configurations [32]. Experiment [30] finds a ${ }^{3} P_{2,1,0}:{ }^{1} P_{1}$ ratio of $0.78: 0.22$; however, this measurement was carried out at $0^{\circ}$ and is thus subject to anisotropic photoelectron angular distribution effects.

In summary, these comparisons of results of our singleconfiguration formulas with both experimental measurements and with results of multiconfiguration calculations indicate the most reliable uses of our formulas for the case of inner $s$ subshells. From Figs. 1 and 2, it appears that the predictions of Eq. (16) of quasiforbidden transitions are quite reliable, as both multiconfiguration calculations and our more general Eq. (15) give very small cross sections for these transitions. Furthermore, a recent

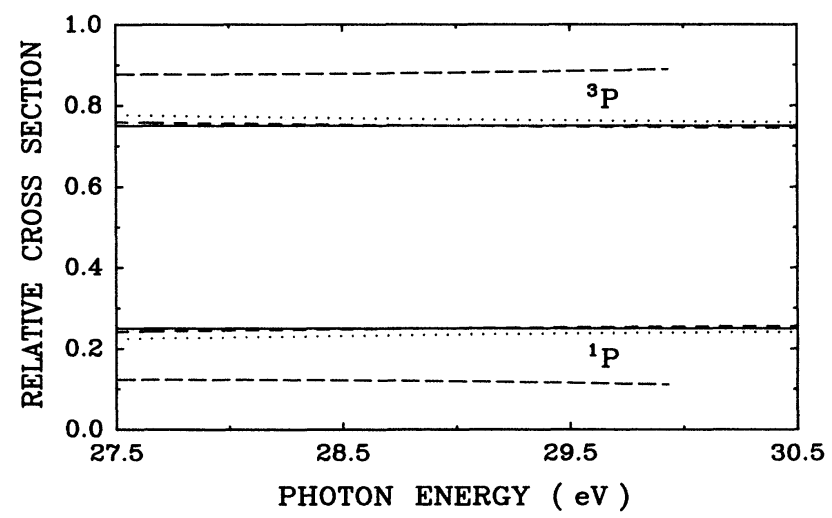

FIG. 3. Relative cross sections for the transitions $\mathrm{Cl} 3 s^{2}$ $3 p^{5}\left({ }^{2} P_{3 / 2}\right)+\gamma \rightarrow \mathrm{Cl} 3 s 3 p^{5}\left({ }^{2 S_{c}+1} P\right)+e^{-}$in three levels of approximation, as specified in Fig. 1. For core spin $S_{c}=1$, the $J_{c}=2,1$, and 0 levels are summed. Predictions of Eqs. (15) and (16) of this paper are identical for the corresponding transitions from the ${ }^{2} P_{1 / 2}$ initial state; the eigenchannel $R$-matrix results are very similar for this case. Note that the results are plotted on a photon energy scale; the photoelectron energies differ by approximately $2.7 \mathrm{eV}$ [34]. experiment [30] did not discern any signal for these transitions. Second, it appears from Figs. 1 and 2 that the relative branching ratios of particular fine-structure transitions, all of which have the same LS quantum numbers, are very accurate, except, as expected, in the vicinity of autoionizing resonances, within which partial cross sections and branching ratios are expected to change rapidly [35]. For the case considered in Figs. 1 and 2, this accuracy is achieved even though configuration mixing is significant. Third, from Fig. 3, it appears that predictions of branching ratios for transitions having different $L S$ quantum numbers must be regarded much more skeptically if significant configuration mixing is suspected, as is known from other studies [9-11].

\section{DISCUSSION AND CONCLUSIONS}

Within a theoretical model of photoionization in which initial and final states are described by single configurations and in which the dynamical amplitudes are evaluated in $L S$ coupling, we have obtained in Eqs. (7) and (10) the partial cross sections $\sigma\left(J_{0}, J_{c}\right)$ corresponding respectively to the two open-shell atom photoionization processes in Eqs. (1) and (2). In contrast to the case of closed-shell atom photoionization, for which the partial cross sections $\sigma\left(J_{0}, J_{c}\right)$ are proportional to $2 J_{c}+1$ [cf. Eq. (11)], one sees from Eqs. (7) and (10) that in general $\sigma\left(J_{0}, J_{c}\right)$ has a complicated dependence on $J_{c}$.

Some aspects of our formulation deserve explicit mention. First, since we presume the dynamical aspects of any calculation are carried out in $L S$ coupling, the small energy differences of the photoionized or detached electrons corresponding to different fine-structure energy levels are not treated explicitly. However, since the dynamical amplitudes do not usually vary much on the scale of typical fine-structure energy differences, these energy effects are expected to be small. Second, it should be pointed out that other coupling schemes can be used to couple $J_{c}, \ell$, and the spin $\left(\frac{1}{2}\right)$ of the photoelectron in the derivation of Eqs. (7) and (10). A change of coupling scheme, however, would not change Eqs. (7) and (10) since all quantum numbers describing the coupling of the photoelectron to the core are summed. Finally, we note that Eqs. (7) and (11) apply not only to photoionization of open-shell atoms and positive ions, but also to photodetachment of open-shell negative ions.

Because of the $L S$ dependence of the dynamical amplitudes in the general Eqs. (7) and (10), the dynamical and geometric factors are tangled together by the summations over $\ell, L$, and $L^{\prime}$. Hence the primary uses of Eqs. (7) and (10) will most likely be to check $a b$ initio theoretical calculations and/or to parametrize experimental data. Only in special cases (such as for closedshell atoms and for photodetachment of $p$-subshell electrons in negative ions) do our general equations involving $L S$-dependent amplitudes reduce to a form that permits completely analytic predictions of relative partial cross sections for fine-structure transitions. These two special cases were treated by Rau and Fano $[4,5]$. 
The simpler term-independent approximation expressions for $\sigma\left(J_{0}, J_{c}\right)$ which we have presented in Eqs. (13) and (14) still retain a complicated dependence on $J_{c}$. However, unlike the more general Eqs. (7) and (10), Eqs. (13) and (14) may be used to calculate the relative intensities of particular fine-structure transitions $J_{0} \rightarrow J_{c}$ for the processes in (1) and (2) completely analytically. This is possible because, once the summations over $L$ and $L^{\prime}$ are performed, the geometric coefficients multiplying the dynamical amplitudes are independent of the photoelectron orbital angular momenta $\ell$, as has been noted by Berkowitz and Goodman [9]. Hence the sum over the dynamical amplitudes becomes disentangled from the geometric factors. Differences of the predicted relative intensities from experimentally measured intensities may be ascribed to dynamical effects such as configuration interaction effects and/or dependence of the radial electron wave functions on the angular momentum quantum numbers $J_{0}, j, J, L$, and $S$.

Note that when the partial cross section for photoionization of a gas sample at a certain temperature resulting in a particular fine-structure level of the residual ion is needed, one can perform a weighted average over the cross sections $\sigma\left(J_{0}, J_{c}\right)$ in Eqs. (7), (10), (13), or (14) according to the statistical distribution of the fine-structure levels $J_{0}$ of the initial state of the system at this temperature.

Finally, in photoionization of inner $s$ subshells of openshell atoms, the approximate Eq. (16) indicates that all fine-structure transitions for which $J_{0}$ and $J_{c}$ differ by more than $\frac{1}{2}$ are forbidden. The more general Eq. (15) indicates, however, that the cross sections for such transi- tions are not necessarily zero. We conclude therefore that such transitions are quasiforbidden and involve strong cancellations among the $L S$-coupling transition amplitudes contributing to the partial cross sections defined by the more general cross section formula in Eq. (15). Our analysis of $\mathrm{Cl} 3 s$-subshell photoionization indicates that such transitions are quasiforbidden even within more complete, multiconfiguration descriptions of the atomic and ionic states. This analysis (cf. Sec. V) also indicates that for $s$ subshells the branching ratio predictions of the formulas presented in this paper can be relied upon most (away from autoionizing regions) wherever the transitions being compared have the same $L S$ quantum numbers and differ only in their fine-structure quantum numbers.

\section{ACKNOWLEDGMENTS}

One of us (A.F.S.) thanks J. Berkowitz, P. C. Engelking, U. Fano, C. H. Greene, W. C. Lineberger, A. R. P. Rau, and F. Robicheaux for helpful discussions. We thank C. D. Caldwell and M. O. Krause for communicating to us their results prior to publication. Finally, we thank F. Robicheaux for providing us with the partial cross sections for particular fine structure transitions for $\mathrm{Cl} 3 s$-subshell photoionization obtained using the eigenchannel $R$-matrix program developed for openshell atom photoionization by $\mathrm{F}$. Robicheaux and C. $\mathrm{H}$. Greene. This work was supported in part by National Science Foundation Grant No. PHY-9108002 and by the Joint Institute for Laboratory Astrophysics.
* Permanent address: Department of Physics and Astronomy, The University of Nebraska-Lincoln, Lincoln, NE 68588-0111.

[1] See, e.g., J. Z. Wu, S. B. Whitfield, C. D. Caldwell, M. O. Krause, P. van der Meulen, and A. Fahlman, Phys. Rev. A 42, 1350 (1990); C. D. Caldwell and M. O. Krause, J. Phys. B 23, 2233 (1990); M. G. Flemming, J. Z. Wu, C. D. Caldwell, and M. O. Krause, Phys. Rev. A 44, 1733 (1991); P. van der Meulen, M. O. Krause, and C. A. de Lange, J. Phys. B 25, 97 (1992).

[2] W. C. Lineberger and B. W. Woodward, Phys. Rev. Lett. 25, 424 (1970).

[3] H. Hotop, T. A. Patterson, and W. C. Lineberger, Phys. Rev. A 8, 762 (1973).

[4] A. R. P. Rau and U. Fano, Phys. Rev. A 4, 1751 (1971).

[5] A. R. P. Rau, in Electron and Photon Interactions with Atoms, edited by H. Kleinpoppen and M. R. C. McDowell (Plenum, New York, 1976), pp. 141-148.

[6] Furthermore, there are errors in the few formulas presented in Ref. [5] for the general case of photoionization. Primarily, the total orbital angular momentum $L$ in Eq. (7) of Ref. [5] is summed incoherently instead of coherently.

[7] P. A. Cox, Structure and Bonding 24, 59 (1975).

[8] P. C. Engelking and W. C. Lineberger, Phys. Rev. A 19,
149 (1979).

[9] J. Berkowitz and G. L. Goodman, J. Chem. Phys. 71, 1754 (1979).

[10] J. Schirmer, L. S. Cederbaum, and J. Kiessling, Phys. Rev. A 22, 2696 (1980).

[11] G. L. Goodman and J. Berkowitz, J. Chem. Phys. 94, 321 (1991).

[12] U. Fano, Comments At. Mol. Phys. 2, 171 (1971).

[13] J. S. Briggs, Rev. Mod. Phys. 43, 189 (1971).

[14] A. F. Starace, in Corpuscles and Radiation in Matter I, edited by W. Mehlhorn, Handbuch der Physik Vol. 31 (Springer, Berlin, 1982). See especially Secs. 2-9.

[15] A. F. Starace, in Ref. [14], Eqs. (9.2) and (9.6).

[16] J. S. Briggs, Ref. [13], p. 214.

[17] A. P. Yutsis, I. B. Levinson, and V. V. Vanagas, The Theory of Angular Momentum (Israel Program for Scientific Translation, Jerusalem, 1962), Eq. (23.7).

[18] A. P. Yutsis, I. B. Levinson, and V. V. Vanagas, Ref. [17], Eq. (A.7.2).

[19] A. P. Yutsis, I. B. Levinson, and V. V. Vanagas, Ref. [17], Eq. (A.6.47).

[20] A. P. Yutsis, I. B. Levinson, and V. V. Vanagas, Ref. [17], Figs. 15.1 and 15.2 .

[21] A. P. Yutsis, I. B. Levinson, and V. V. Vanagas, Ref. [17], Eq. (17.2). 
[22] A. F. Starace, in Ref. [14], Eqs. (9.2) and (9.7).

[23] E. P. Wigner, Phys. Rev. 73, 1002 (1948).

[24] A. P. Yutsis, I. B. Levinson, and V. V. Vanagas, Ref. [17], Eq. (19.3).

[25] A. P. Yutsis, I. B. Levinson, and V. V. Vanagas, Ref. [17], pp. 63 and 64 .

[26] A. R. P. Rau, in Ref. [5], Eq. (4), where Rau's quantum numbers $J^{\prime}, j_{c}$, and $J$ correspond to our $J_{0}, J_{c}$, and $\nu$. Note that our Eq. (12) does not have the factor $2 J^{\prime}+1$ ( $\left[J_{0}\right]$ in our notation) included in Rau's Eq. (4). This factor was canceled when we averaged over initial-state magnetic quantum numbers $M_{0}$ [cf. our Eq. (3)].

[27] A. P. Yutsis, I. B. Levinson, and V. V. Vanagas, Ref. [17], Eq. (A.6.1).

[28] Specifically, our Eq. (13) corresponds directly with Eq. (29) of Ref. [7], with the results in the Appendix of Ref. [8], with Eq. (37) of Ref. [10] as well as indirectly with the intermediate coupling results in Eq. (13) of Ref. [9] and in Eq. (14) of Ref. [11]. Note that all of these other results are expressed in terms of a sum over products of $9 j$ coefficients. For the single configuration, $L S$-coupling case we consider here the sum over a squared $9 j$ coefficient is an alternative way of representing the same $12 j$ coefficient that we represent as a sum over a product of squared $6 j$ coefficients. Our expression is simpler since in practice $6 j$ coefficients are easier to evaluate numerically than $9 j$ coefficients.

[29] A. P. Yutsis, I. B. Levinson, and V. V. Vanagas, Ref. [17], Fig. 20.3.

[30] M. O. Krause, C. D. Caldwell, S. B. Whitfield, C. A. de Lange, and P. van der Meulen (unpublished).

[31] F. Robicheaux and C. H. Greene, Phys. Rev. A 46, 3821 (1992).

[32] F. Robicheaux and C. H. Greene, Phys. Rev. A (to be published); F. Robicheaux (private communication).

[33] Substituting Eq. (8) for the $15 j$ symbol in either Eq. (7) or Eq. (10) permits one to carry out a summation over $J_{c}$ using Eq. (A.6.18) of Ref. [17]. This results in the dummy index $a$ in Eq. (8) being zero, which collapses the sum over Eqs. (7) and (10) to much simpler expressions that are independent of $J_{0}$.

[34] Note that in our calculations we have used an average threshold excitation energy for the ${ }^{3} P$ ionic states of $24.633 \mathrm{eV}$, which is obtained by weighting the three finestructure thresholds by their multiplicity. The ${ }^{1} P$ ionic state threshold energy used is $27.357 \mathrm{eV}$. All of these values were obtained using the tabulated energies in C. E. Moore, Atomic Energy Levels, Natl. Bur. Stand. (U.S.) Circ. No. 467 (U.S. GPO, Washington, D.C., 1949), Vol. I.

[35] A. F. Starace, Phys. Rev. A 16, 231 (1977). 